Research Article

\title{
Solving the Single-Valued Trapezoidal Neutrosophic Transportation Problems through the Novel Dhouib-Matrix-TP1 Heuristic
}

\author{
Souhail Dhouib \\ OLID Laboratory, Higher Institute of Industrial Management, University of Sfax, Sfax, Tunisia \\ Correspondence should be addressed to Souhail Dhouib; souh.dhou@gmail.com
}

Received 12 August 2021; Revised 19 October 2021; Accepted 21 October 2021; Published 2 November 2021

Academic Editor: Ewa Rak

Copyright (c) 2021 Souhail Dhouib. This is an open access article distributed under the Creative Commons Attribution License, which permits unrestricted use, distribution, and reproduction in any medium, provided the original work is properly cited.

\begin{abstract}
The transportation problem has been widely studied in the field of supply chain management where circulation of products with a minimal transportation cost is an important issue. This paper presents the first adaptation of the Dhouib-Matrix-TP1 heuristic to solve the transportation problem in single-valued trapezoidal neutrosophic environment. Hence, the recently developed DhouibMatrix-TP1 heuristic is enriched with two functions to solve the neutrosophic transportation problem. On the one hand, a defuzzification function is exploited in order to convert the single-valued trapezoidal neutrosophic numbers to crisp numbers. On the other hand, an original metric function (Average-Min) is proposed with the intention of performing the nodes selection process. With an illustration from a literature example, we show to the decision maker the multiple advantages of the novel heuristic Dhouib-Matrix-TP1 which can be easily implemented in real-life industrial transportation under neutrosophic environment.
\end{abstract}

\section{Introduction}

The neutrosophic concept was introduced in 1995 by Smarandach [1] in order to make the chance of emulating the human thinking by using at the same time three membership functions: the Truth, the Falsity, and the Indeterminacy, noted, respectively, as $(T),(F)$, and $(I)$. These three membership functions compose the neutrosophic set $\langle T, I, F\rangle$, where $T, I$ and $F \in[0,1]$.

The neutrosophic concept is becoming a major focus of several research papers. In fact, Ibrahem et al. [2] projected the idea of applying the neutrosophic analytical hierarchy process model to forecast the default of clients using five financial criteria ( $C \_1$ : the working capital, $C \_2$ : the Liquidity, C_3: the Profitability, $C_{-} 4$ : the Costs, and C_5: the Customer obligation). The information was gathered in the form of neutrosophic datasets and evaluated in the credit department of one of private banks. In [3], the generalized assignment problem is considered in neutrosophic set theory, where elements of the cost matrix are presented as trapezoidal fuzzy neutrosophic elements, and then, the problem is solved by the zero-suffix method. In [4], the assignment problem with costs as nonagonal number is optimized under three domains: fuzzy, intuitionistic, and neutrosophic. In [5], the neutrosophic assignment problem with the pentagonal neutrosophic number is solved using a new technique for ranking neutrosophic numbers into real numbers by the means of the magnitude function. In [6], the multicriteria assignment problem is solved using two methods where the different criteria have been considered as neutrosophic elements.

In [7], the order relation technique is applied to optimize the neutrosophic trapezoidal fuzzy assignment problem. In [8], the Branch and Bound method is used to optimize the neutrosophic assignment problem, where elements of the matrix are triangular fuzzy numbers. In $[9,10]$, the Branch and Bound technique and the Ones Assignment methods are, respectively, used to solve the neutrosophic Travelling Salesman Problem. Sikkannanl and Shanmugavel [11] introduce a new method to optimize the neutrosophic fuzzy 
transportation problem using the Mean and Complete Contingency Cost Table.

The idea of trapezoidal neutrosophic is considered in [12], and the triangular fuzzy neutrosophic sets are proposed in [13]. Also, the Travelling Salesman Problem is solved under single-valued triangular neutrosophic parameters in [14]. A real-life TP under neutrosophic domain is solved in $[15,16]$, while the neutrosophic shortest path problem is optimized in [17]. Several operations on neutrosophic matrices are introduced in [18], and an application of the multicriteria group decision in a real-life problem is illustrated. The application of the neutrosophic N-structures to p-ideals of BCI-algebras is presented in [19]. The optimal hydrogen power plant site using a single-valued neutrosophic multiattribute decision-making technique is selected in [20].

In this paper, we focus to practically help the decision maker to handle a suitable solution for TP in neutrosophic domains and specially the case of single-valued trapezoidal numbers. Obviously, an easy and convivially neutrosophic decision support system is needed in order to depict graphically the crisp and neutrosophic solutions (graphical representation of the crisp and the single-valued trapezoidal neutrosophic solutions). Furthermore, this paper presents the first application of our novel heuristic Dhouib-MatrixTP1 (DM-TP1) to solve the TP under the single-valued trapezoidal neutrosophic environment. The DM-TP1 was developed using Python programming language and enhanced with two techniques: on the one hand, the defuzzification score function in order to convert the neutrosophic trapezoidal fuzzy number to crisp number, and on the other hand, by the proposed original metric function (Average-Min) in order to drive the node (sources and destinations) selection process.

The remaining of this paper is organized as follows. Section 2 introduces the transportation problem. Section 3 presents some basic definitions on neutrosophic environment. Section 4 explains in detail the proposed heuristic DM-TP1. Section 5 depicts the stepwise application of the proposed method. Finally, conclusions with further research works are presented in Section 6.

\section{The Transportation Problem}

The transportation problem (TP) is a very well-studied topic in the field of supply chain management, and it deals with the minimization of the transportation cost of products from a certain number of sources to a certain number of destinations. The objective of the TP is to search for the optimal value of $x_{i j}$ that will minimize the total transportation cost (see equation (1)) while satisfying the supply and demand restrictions (see equation (2)). The TP is mathematically formulated as follows.

Minimize

$$
Z=\sum_{i=1}^{m} \sum_{j=1}^{n} c_{i j} x_{i j}
$$

which subjects to

$$
\begin{gathered}
\sum_{j=1}^{n} x_{i j}=a_{i} ; i=1,2, \ldots, m, \\
\sum_{i=1}^{m} x_{i j}=b_{j} ; j=1,2, \ldots, n, \\
x_{i j} \geq 0 \text { for all } i \text { and } j .
\end{gathered}
$$

The notation of the TP is

$m$ is the total number of supplies (sources)

$n$ is the total number of demands (destinations)

$a_{i}$ is the amount of supply at source $i$

$b_{j}$ is the amount of demand at destination $j$

$c_{i j}$ is the transportation cost from supply $i$ to demand $j$

$x_{i j}$ is the amount to be shipped from source $i$ to destination $j$

The TP was firstly designed by Hitchcock in [21]. Charnes and Cooper in [22] proposed the Stepping Stone Method to find the optimal solution for TP. Dantzig, in [23], presented the simplex technique for the classical TP. Shell, in [24], introduced a solid transportation problem using three bounds: supply, demand, and conveyance that represent the different transport modes such as ships, goods train, trucks, and cargo flights. Haley, in [25], presented an enhanced version of the modified distribution method to optimize the solid transportation problem.

In real-life situations and in many cases, the decision maker has no precise information about the TP parameters: the transportation costs and the value of demands and supplies. In this situation, the corresponding elements defining the problem can be formulated by the means of fuzzy set, intuitionistic set, or neutrosophic set. Pandian and Natarajan, in [26], solved the TP under trapezoidal fuzzy numbers for all parameters. Kumar, in [27], designed the PSK method to solve the fuzzy TP type-1 and type-3. Mhaske and Bondar, in [28], solved the TP under triangular, pentagonal, and heptagonal fuzzy numbers and proposed the application of Lagrange's polynomial function for the nonagonal and hendecagonal fuzzy numbers. Dinagar and Thiripurasundari, in [29], solved the fuzzy TP under intuitionistic trapezoidal fuzzy numbers, and Sikkannan and Shanmugavel, in [30], used the magnitude ranking function with the Entire Contingency Cost Table method to solve the triangular fuzzy TP.

\section{Basic Definitions}

The neutrosophic concept allows to the decision maker, in real-world problems, to rely not only on true values but also on false ones as well as on indeterminacy membership.

Definition 1 (see [1]). Let us define by $X$ the space of objects and define $x$ as its generic element $x \in X$. The neutrosophic set $N$ has the form $N=\left\{\left\langle x: T_{N}(x), I_{N}(x), F_{N}(x)\right\rangle, x \in X\right\}$, where the functions $T, I, F: X \longrightarrow]^{-} 0,1^{+}[$with the condition ${ }^{-} 0 \leq T_{N}(x)+I_{N}(x)+F_{N}(x) \leq 3^{+}$. 
Definition 2 (see [31]). The truth (T), indeterminacy $(I)$, and falsity $(F)$ membership functions for the neutrosophic trapezoidal fuzzy $N=\left\langle\left(N_{a}, N_{b}, N_{c}, N_{d}\right) ; T_{N}, I_{N}, F_{N}\right\rangle$ number are defined by

$$
\mu(X)= \begin{cases}\frac{(x-a) T_{N}}{b-a}, & a \leq x \leq b, \\ T_{N} & b \leq x \leq c, \\ \frac{(d-x) T_{N}}{d-c}, & c \leq x \leq d, \\ 0, & \text { otherwise, }\end{cases}
$$

$\theta(X)= \begin{cases}\frac{b-x+(x-a) I_{N}}{b-a}, & a \leq x \leq b, \\ I_{N} & b \leq x \leq c, \\ \frac{x-c(d-x) I_{N}}{d-c}, & c \leq x \leq d, \\ 0, & \text { otherwise, }\end{cases}$

$$
\lambda(X)= \begin{cases}\frac{b-x+(x-a) F_{N}}{b-a}, & a \leq x \leq b, \\ F_{N}, & b \leq x \leq c, \\ \frac{x-c+(d-x) F_{N},}{d-c}, & c \leq x \leq d, \\ 0 & \text { otherwise. }\end{cases}
$$

Here is an example (see Figure 1) of a graphical representation of a single-valued trapezoidal neutrosophic set $N=\langle(1,3,5,7) ; 0.9,0.2,0.4\rangle$.

Definition 3 (see [15]). Let us assume two neutrosophic trapezoidal fuzzy numbers $N=\left\langle\left(N_{a}, N_{b}, N_{c}, N_{d}\right) ; T_{N}, I_{N}\right.$ ,$\left.F_{N}\right\rangle$ and $M=\left\langle\left(M_{a}, M_{b}, M_{c}, M_{d}\right) ; T_{M}, I_{M}, F_{M}\right\rangle$. Then, their operations are defined as follows: 


$$
\begin{aligned}
& M+N=\left\langle\left(M_{a}+N_{a}, M_{b}+N_{b}, M_{c}+N_{c}, M_{d}+N_{d}\right) ; T_{M} \wedge T_{N}, I_{M} \vee I_{N}, F_{M} \vee F_{N}\right\rangle, \\
& M-N=\left\langle\left(M_{a}-N_{d}, M_{b}-N_{c}, M_{c}-N_{b}, M_{d}-N_{a}\right) ; T_{M} \wedge T_{N}, I_{M} \vee I_{N}, F_{M} \vee F_{N}\right\rangle, \\
& M \times N= \begin{cases}\left\langle\left(M_{a} \times N_{a}, M_{b} \times N_{b}, M_{c} \times N_{c}, M_{d} \times N_{d}\right) ; T_{M} \wedge T_{N}, I_{M} \vee I_{N}, F_{M} \vee F_{N}\right\rangle \text { if } & M_{d}>0, N_{d}>0, \\
\left\langle\left(M_{a} \times N_{d}, M_{b} \times N_{c}, M_{c} \times N_{b}, M_{d} \times N_{a}\right) ; T_{M} \wedge T_{N}, I_{M} \vee I_{N}, F_{M} \vee F_{N}\right\rangle \text { if } & M_{d} \prec 0, N_{d}>0, \\
\left\langle\left(M_{d} \times N_{d}, M_{c} \times N_{c}, M_{b} \times N_{b}, M_{a} \times N_{a}\right) ; T_{M} \wedge T_{N}, I_{M} \vee I_{N}, F_{M} \vee F_{N}\right\rangle \text { if } & M_{d} \prec 0, N_{d} \prec 0,\end{cases} \\
& \frac{M}{N}=\left\{\begin{array}{l}
\left.\left\langle\left(\frac{M_{a}}{N_{d}}, \frac{M_{b}}{N_{c}}, \frac{M_{c}}{N_{b}}, \frac{M_{d}}{N_{a}}\right) ; T_{M} \wedge T_{N}, I_{M} \vee I_{N}, F_{M} \vee F_{N}\right\rangle \text { if } M_{d}\right\rangle\left\langle 0, N_{d}>0,\right. \\
\left\langle\left(\frac{M_{d}}{N_{d}}, \frac{M_{c}}{N_{c}}, \frac{M_{b}}{N_{b}}, \frac{M_{a}}{N_{a}}\right) ; T_{M} \wedge T_{N}, I_{M} \vee I_{N}, F_{M} \vee F_{N}\right\rangle \text { if } \quad M_{d} \prec 0, N_{d}>0, \\
\left\langle\left(\frac{M_{d}}{N_{a}}, \frac{M_{c}}{N_{b}}, \frac{M_{b}}{N_{c}}, \frac{M_{a}}{N_{d}}\right) ; T_{M} \wedge T_{N}, I_{M} \vee I_{N}, F_{M} \vee F_{N}\right\rangle \text { if } \quad M_{d} \prec 0, N_{d} \prec 0,
\end{array}\right. \\
& k * N= \begin{cases}\left\langle\left(k * N_{a}, k * N_{b}, k * N_{c}, k * N_{d}\right) ; T_{N}, I_{N}, F_{N}\right\rangle \text { if } & k>0, \\
\left\langle\left(k * N_{d}, k * N_{c}, k * N_{b}, k * N_{a}\right) ; T_{N}, I_{N}, F_{N}\right\rangle \text { if } & k \prec 0,\end{cases} \\
& N^{-1}=\left\langle\left(\frac{1}{N_{d}}, \frac{1}{N_{c}}, \frac{1}{N_{b}}, \frac{1}{N_{a}}\right) ; T_{M}, I_{M}, F_{M}\right\rangle \text {, where } \quad N \neq 0 .
\end{aligned}
$$

Definition 4 (see [16]). For a single-valued neutrosophic number $N=\left\langle\left(N_{a}, N_{b}, N_{c}, N_{d}\right) ; T_{N}, I_{N}, F_{N}\right\rangle$, its score function is defined by $M\left(\widetilde{c}_{i j}^{N}\right)$ as follows:

$$
\begin{aligned}
M\left(\widetilde{c}_{i j}^{N}\right)= & \left(\min _{1 \leq i \leq n}\left(\mu_{c_{i j}^{N}}\right)+\min _{1 \leq i \leq n}\left(\mathcal{\vartheta}_{c_{i j}^{N}}\right)+\max _{1 \leq i \leq n}\left(1-\lambda \widetilde{c}_{i j}^{N}\right)\right) \\
& \times \sum_{i=1}^{m} \sum_{j=1}^{n}\left(\frac{S\left(\widetilde{c}_{i j}^{N} x_{i j}\right)}{\mu_{c_{i j}^{N}}+\left(1-\mathcal{\vartheta}_{c_{i j}^{N}}\right)+\left(1-\lambda_{c_{i j}}\right)}\right),
\end{aligned}
$$

where

$$
\begin{aligned}
S\left(\widetilde{c}_{i j}^{N}\right)= & \left(\frac{1}{16}\right) \times\left(N_{a}+N_{b}+N_{c}+N_{d}\right) \\
& \times\left(T_{N}+\left(1-I_{N}\right)+\left(1-F_{N}\right)\right) .
\end{aligned}
$$

\section{The Proposed Method: Dhouib-Matrix- TP1 (DM-TP1)}

Very recently, we invent in [32] a new constructive method entitled Dhouib-Matrix-TSP1 (DM-TSP1) to solve the classical Travelling Salesman Problem (TSP). The proposed method is based on several rules, and we handle it to solve the trapezoidal fuzzy TSP using the magnitude ranking function [33]. Then, in [34], we enhance the DM-TSP1 method with the $\alpha$-Cut Technique to optimize the octagonal fuzzy TSP. Moreover, in [35], we generate a stochastic version, namely, the Dhouib-Matrix-TSP2 to solve the TSP.
In [36], we introduce a novel heuristic entitled DhouibMatrix-TP1 (DM-TP1) to solve the classical transportation problem (TP).

In this paper, we focus on the application of enhanced version of the DM-TP1 to solve the neutrosophic trapezoidal fuzzy TP. In fact, the DM-TP1 was presented in [18] using the standard deviation metric, whereas in this paper, we introduce a new original metric the (Average-Min) metric in order to drive the selection of sources and destinations. A stepwise application of this metric will be presented in the next section.

Furthermore, we enrich the DM-TP1 with the defuzzification score function [16] in order to convert the neutrosophic trapezoidal fuzzy parameters to crisp number. Thus, the score function for the neutrosophic trapezoidal fuzzy number $N=\left\langle\left(N_{a}, N_{b}, N_{c}, N_{d}\right) ; T_{N}, I_{N}, F_{N}\right\rangle$ is defined by equations (5) and (6).

The proposed method DM-TP1 is accomplished into nine steps (see Figure 2). Steps 1, 2, and 9 are executed only once, and Steps 3, 4, 5, 6, 7, and 8 are repeated until all columns are discarded.

Step 1 : transform the single-valued trapezoidal neutrosophic parameters to crisp parameters using the defuzzification functions described in equations (5) and (6).

Step 2 : balance the sum of supplies and demands for the transport matrix by adding fictive row and column with corresponding quantity. Next, in the transportation matrix, we add a row below, 
entitled the Average Min Demand Column (AMDC), and insert a column at right, named the Average Min Supply Row (AMSR).

Step 3 : compute the original function (Average-Min) for each row which affects the corresponding value for each element in the AMSR.

Step 4 : apply the same operation on each column. Compute the original proposed function $(\mathrm{Av}$ erage-Min) for each column. Then, it affects the corresponding value for each element of the AMDC.

Step 5 : identify the highest element among the AMSR and AMDC; if it is in AMSR, then select the minimal element $\left(x_{i j}\right)$ of its corresponding row else check the minimal element $\left(x_{i j}\right)$ of its corresponding column.

Step 6 : if $a_{i} \leq b_{j}$ then allocate the $a_{i}$ amount of units to $x_{i j}$, which affects $b_{j}^{\prime}=b_{j}-a_{i}$, and discard row $i$.

Step 7 : if $a_{i}>b_{i}$ then allocate the $b_{j}$ amount of units to $x_{i j}$, which affects $a_{i}^{\prime}=a_{i}-b_{j}$, and discard column $j$.

Step 8 : repeat Steps 3, 4, 5, 6, and 7 until all columns are discarded.

Step 9 : calculate the total minimal transportation cost.

\section{Numerical Example}

In this section, an example from [16] is used to prove the performance of the DM-TP1 heuristic in order to easily solve the TP under single-valued trapezoidal neutrosophic numbers. In fact, this problem was firstly introduced by [15] in order to present a peanut butter manufacturing company with three sources and four different destinations. In this problem, the cost parameters $\left(\tilde{c}_{i j}^{N}\right)$ are presented as singlevalued trapezoidal neutrosophic numbers, while the supply and demand quantities are presented as crisp numbers as given in Figure 3. below:

The mathematical formulation of this problem is given

$$
\begin{aligned}
& \text { Minimize }: \widetilde{Z}^{N}=\sum_{i=1}^{m} \sum_{j=1}^{n} \widetilde{c}_{i j}^{N} x_{i j} \text {, } \\
& \text { Subject to : } \\
& \sum_{j=1}^{n} x_{i j}=a_{i} ; \quad i=1,2, \ldots, m, \\
& \sum_{i=1}^{m} x_{i j}=b_{j} ; \quad j=1,2, \ldots, n, \\
& x_{i j} \geq 0 \text { for all } i \text { and } j .
\end{aligned}
$$

The DM-TP1 starts by transforming the neutrosophic trapezoidal fuzzy number to crisp number using equation (5) and equation (6).

Here is an example for the first cost element: $N=\langle(3,5,6,8) ; 0.6,0.5,0.4\rangle$.
Then, $S(N)=(1 / 16) \times(3+5+6+8) \times(2+0.6-0.5-$ $0.4)=2.3$.

The second step is to calculate the AMSR and the AMDC for all rows and columns using the proposed formula (Average-Min). Let us give an example how to compute easily the first element of AMSR:

$$
\operatorname{AMSR}[1]=\text { Average }_{r 1}-\operatorname{Min}_{r 1} \text {, }
$$

where

$$
\begin{gathered}
\text { Average }_{r 1}=\frac{(3+4+8+9)}{4}=6, \\
\operatorname{Min}_{r 1}=(3,4,8,9)=3 .
\end{gathered}
$$

Thus, $\operatorname{AMSR}[1]=6-3=3$.

The same process is followed for the first element for AMDC:

$$
\operatorname{AMDC}[1]=\text { Average }_{c 1}-\operatorname{Min}_{c 1},
$$

where

$$
\begin{aligned}
\text { Average }_{c 1} & =\frac{(3+1+4)}{3}=2.66, \\
\operatorname{Min}_{c 1} & =(3,1,4)=1 .
\end{aligned}
$$

Thus, $\mathrm{AMDC}[1]=2.66-1=1.66$.

The next step is to select the highest element in AMSR and AMDC: 3.75 is the highest value and it is in the second row of AMSR (see Figure 4).

Thus, select the minimal element in the second row of AMSR which is equal to 1 at position $d_{21}$, affect 17 units (which represents the smallest element between demand 17 and supply 24), and discard the saturated element which is column 1. Hence, compute again the AMSR and AMDC indicators, select the highest one (3.33), and find its corresponding minimal element (3) at position $d_{32}$ (see Figure 5).

Therefore, 28 units (the smallest element between demand 28 and supply 30 ) are affected and the saturated element which is column 3 is discarded. Next, calculate the AMSR and AMDC indicators, select the highest one (2.50), and find its corresponding minimal element (4) at position $d_{12}$ (see Figure 6).

Consequently, 23 units (the smallest element between demand 23 and supply 26) are affected and the saturated element which is column 2 is discarded. Next, calculate the AMSR and AMDC indicators, select the highest one (1.67), and find its corresponding minimal element (5) at position $d_{34}$ (see Figure 7).

Accordingly, 2 units (the smallest element between demand 12 and supply 2) are affected and the saturated element which is row 3 is discarded. Next, calculate the AMSR and AMDC indicators, select the highest one (1.50), and find its corresponding minimal element (6) at position $d_{24}$ (see Figure 8 ).

Hence, 7 units (the smallest element between demand 10 and supply 7) are affected and the saturated element which is 


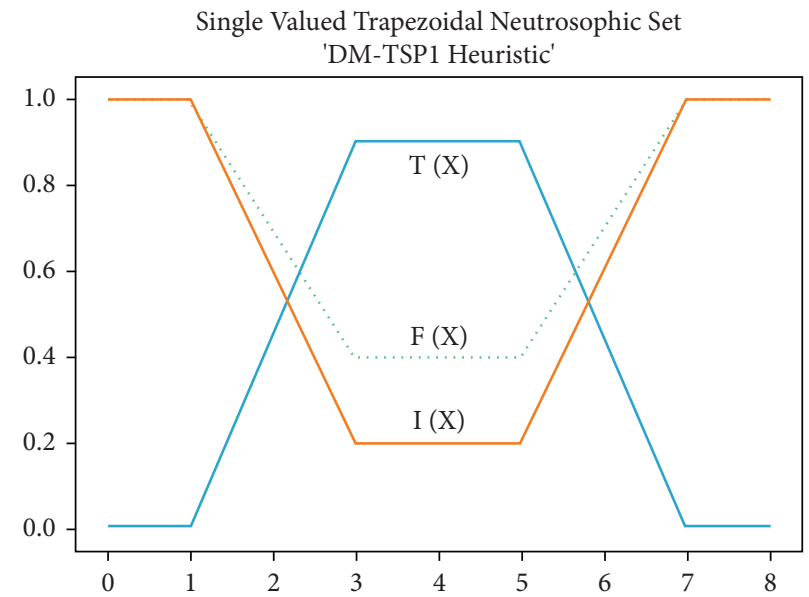

FIGURE 1: Graphical representation of a single-valued trapezoidal neutrosophic set.

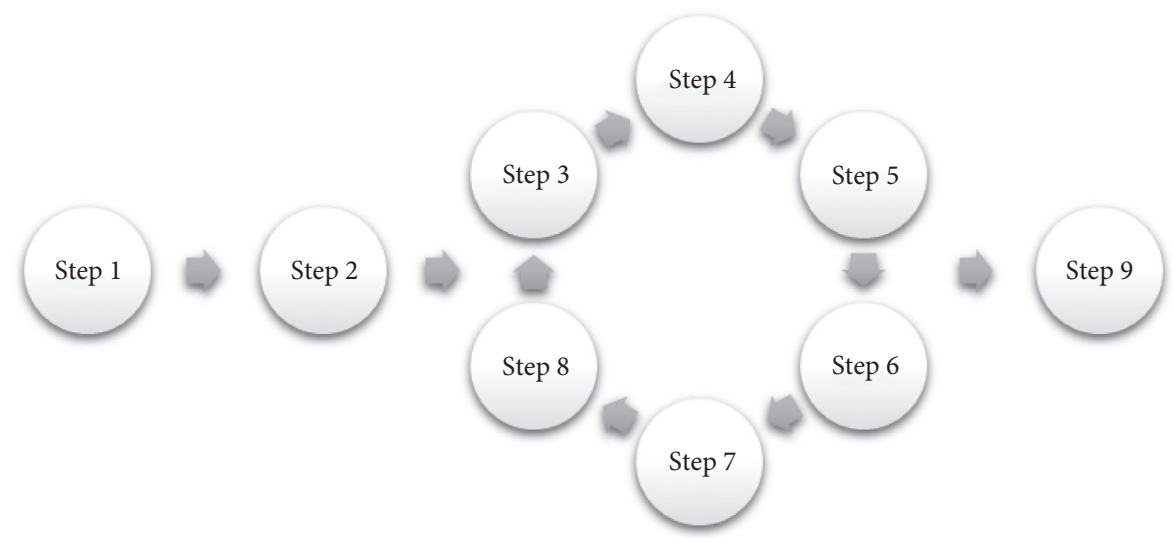

FIgure 2: The flowchart of the proposed DM-TP1.

\begin{tabular}{|c|c|c|c|c|c|}
\hline \multirow{2}{*}{ Source } & \multicolumn{4}{|c|}{ Destination } & \multirow{2}{*}{ Inventory } \\
\hline & D1 & D2 & D3 & D4 & \\
\hline O1 & $\begin{array}{c}(3,5,6,8) \\
0.6,0.5,0.4\end{array}$ & $\begin{array}{c}(5,8,10,14) ; \\
0.3,0.6,0.6\end{array}$ & $\begin{array}{c}(12,15,19,22) \\
0.6,0.4,0.5\end{array}$ & $\begin{array}{c}(14,17,21,28) ; \\
0.8,0.2,0.6\end{array}$ & 26 \\
\hline $\mathrm{O} 2$ & $\begin{array}{c}(0,1,3,6) \\
0.7,0.5,0.3\end{array}$ & $\begin{array}{l}(5,7,9,11) ; \\
0.9,0.7,0.5\end{array}$ & $\begin{array}{c}(15,17,19,22) \\
0.4,0.8,0.4\end{array}$ & $\begin{array}{c}(9,11,14,16) \\
0.5,0.4,0.7\end{array}$ & 24 \\
\hline O3 & $\begin{array}{c}(4,8,11,15) \\
0.6,0.3,0.2\end{array}$ & $\begin{array}{c}(1,3,4,6) ; \\
0.6,0.3,0.5\end{array}$ & $\begin{array}{l}(5,7,8,10) ; \\
0.5,0.4,0.7\end{array}$ & $\begin{array}{c}(5,9,14,19) \\
0.3,0.7,0.6\end{array}$ & 30 \\
\hline Demand & 17 & 23 & 28 & 12 & \\
\hline
\end{tabular}

FIGURE 3: The single-valued trapezoidal neutrosophic TP.

row 2 is discarded. Finally, it remains only one element at position $d_{14}$, affects 3 units, and discards row 1 (see Figure 9).

Therefore, the obtained optimal solution using DM-TP1 heuristic is $x_{14}=3, x_{24}=7, x_{34}=2, x_{12}=23, x_{33}=28$, and $x_{21}=17$ with $Z=(9 * 3)+(6 * 7)+(5 * 2)+(4 * 23)$ $+(3 * 28)+(1 * 17)=272$.
Then, the DM-TP1 heuristic found the minimal total crisp cost equal to 272 and graphically presented in Figure 10. This result was also found by [16].

Consequently, the minimal total single-valued trapezoidal neutrosophic cost is 


\begin{tabular}{|c|c|c|c|c|c|c|}
\hline \multirow{2}{*}{ Source } & \multicolumn{4}{|c|}{ Destination } & \multirow{2}{*}{ Inventory } & \multirow{2}{*}{ AMSR } \\
\hline & $D 1$ & D2 & D3 & D4 & & \\
\hline D1 & 3 & 4 & 8 & 9 & 26 & 3.00 \\
\hline$D 2$ & 1 & 4 & 8 & 6 & 24 & 3.75 \\
\hline D3 & 4 & 2 & 3 & 5 & 30 & 1.50 \\
\hline Demand & 17 & 23 & 28 & 12 & & \\
\hline AMDC & 1.66 & 1.33 & 3.33 & 1.67 & & \\
\hline
\end{tabular}

FIgURE 4: After the defuzzification step.

\begin{tabular}{|c|c|c|c|c|c|c|}
\hline \multirow{2}{*}{ Source } & \multicolumn{4}{|c|}{ Destination } & \multirow{2}{*}{ Inventory } & \multirow{2}{*}{ AMSR } \\
\hline & $D 1$ & D2 & D3 & D4 & & \\
\hline D1 & & 4 & 8 & 9 & 26 & 3.00 \\
\hline D2 & & 4 & 8 & 6 & 7 & 3.00 \\
\hline D3 & & 2 & 3 & 5 & 30 & 1.33 \\
\hline Demand & & 23 & 28 & 12 & & \\
\hline AMDC & & 1.33 & 3.33 & 1.67 & & \\
\hline
\end{tabular}

FIgURE 5: Discard column 1.

$$
\begin{aligned}
\text { Minimize: } \widetilde{Z}^{N}= & \sum_{i=1}^{3} \sum_{j=1}^{4} \widetilde{c}_{i j}^{N} x_{i j}, \\
\widetilde{Z}^{N}= & 3 \times\langle(14,17,21,28) ; 0.3,0.8,0.7\rangle, \\
& +7 \times\langle(9,11,14,16) ; 0.3,0.8,0.7\rangle, \\
& +2 \times\langle(5,9,14,19) ; 0.3,0.8,0.7\rangle, \\
& +23 \times\langle(5,8,10,14) ; 0.3,0.8,0.7\rangle, \\
& +28 \times\langle(5,7,8,10) ; 0.3,0.8,0.7\rangle, \\
& +17 \times\langle(0,1,3,6) ; 0.3,0.8,0.7\rangle, \\
= & \langle(370,543,694,938) ; 0.3,0.8,0.7\rangle .
\end{aligned}
$$

The graphical representation of the minimal total solution is depicted in Figure 11, where the total single-valued trapezoidal neutrosophic optimal solution will be greater than 370 and less than 938 with a level of acceptance $30 \%$ for the total neutrosophic cost lying between 543 and 694 .

Therefore, a decision maker can conclude the minimal trapezoidal neutrosophic cost from the range 370 to 938 , with its truth, indeterminacy, and falsity degrees. The truth membership function for the generated solution is denoted by

$$
\mu(X)= \begin{cases}\frac{(x-370) \times 0.3}{543-370}, & 370 \leq x \leq 543, \\ 0.3, & 543 \leq x \leq 694 \\ \frac{(938-x) \times 0.3}{938-694}, & 694 \leq x \leq 938, \\ 0, & \text { otherwise. }\end{cases}
$$

Correspondingly, the indeterminacy membership function for the minimal single-valued trapezoidal neutrosophic number cost is presented by

$$
\vartheta(X)= \begin{cases}\frac{543-x+(x-370) \times 0.8}{543-370}, & 370 \leq x \leq 543, \\ 0.8, & 543 \leq x \leq 694, \\ \frac{x-694+(938-x) \times 0.8}{938-694} & 694 \leq x \leq 938, \\ 0, & \text { otherwise. }\end{cases}
$$




\begin{tabular}{|c|c|c|c|c|c|c|}
\hline \multirow{2}{*}{ Source } & \multicolumn{4}{|c|}{ Destination } & \multirow{2}{*}{ Inventory } & \multirow{2}{*}{ AMSR } \\
\hline & D1 & D2 & D3 & D4 & & \\
\hline D1 & & 4 & & 9 & 26 & 2.50 \\
\hline$D 2$ & & 4 & & 6 & 7 & 1.00 \\
\hline D3 & & 2 & & 5 & 2 & 1.50 \\
\hline Demand & & 23 & & 12 & & \\
\hline AMDC & & 1.33 & & 1.67 & & \\
\hline
\end{tabular}

FIgURE 6: Discard column 3.

\begin{tabular}{|c|c|c|c|c|c|c|}
\hline \multirow{2}{*}{ Source } & \multicolumn{4}{|c|}{ Destination } & \multirow{2}{*}{ Inventory } & \multirow{2}{*}{ AMSR } \\
\hline & $D 1$ & D2 & D3 & $D 4$ & & \\
\hline D1 & & & & 9 & 3 & 0.00 \\
\hline D2 & & & & 6 & 7 & 0.00 \\
\hline D3 & & & & 5 & 2 & 0.00 \\
\hline Demand & & & & 12 & & \\
\hline AMDC & & & & 1.67 & & \\
\hline
\end{tabular}

Figure 7: Discard column 2.

\begin{tabular}{|c|c|c|c|c|c|c|}
\hline \multirow{2}{*}{ Source } & \multicolumn{4}{|c|}{ Destination } & \multirow{2}{*}{ Inventory } & \multirow{2}{*}{ AMSR } \\
\hline & D1 & D2 & D3 & D4 & & \\
\hline$D 1$ & & & & 9 & 3 & 0.00 \\
\hline D2 & & & & 6 & 7 & 0.00 \\
\hline \multicolumn{7}{|l|}{ D3 } \\
\hline Demand & & & & 10 & & \\
\hline AMDC & & & & 1.50 & & \\
\hline
\end{tabular}

Figure 8: Discard row 3.

\begin{tabular}{|c|c|c|c|c|c|c|}
\hline \multirow{2}{*}{ Source } & \multicolumn{4}{|c|}{ Destination } & \multirow{2}{*}{ Inventory } & \multirow{2}{*}{ AMSR } \\
\hline & $D 1$ & D2 & D3 & $D 4$ & & \\
\hline$D 1$ & & & & 9 & 3 & 0.00 \\
\hline \multicolumn{7}{|l|}{ D2 } \\
\hline \multicolumn{7}{|l|}{ D3 } \\
\hline Demand & & & & 3 & & \\
\hline AMDC & & & & 0.00 & & \\
\hline
\end{tabular}

Figure 9: Discard row 2. 


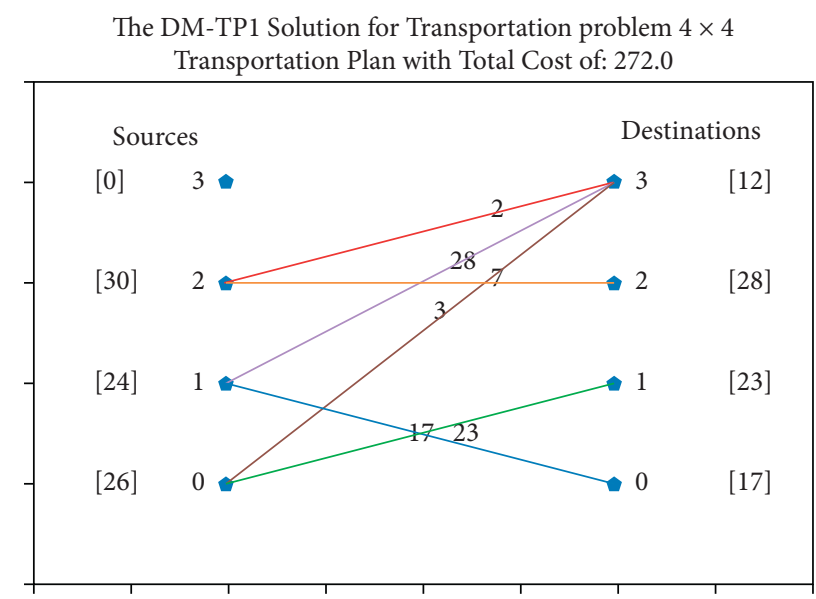

FIGURE 10: The generated transportation problem network diagram plan using DM-TP1 heuristic.

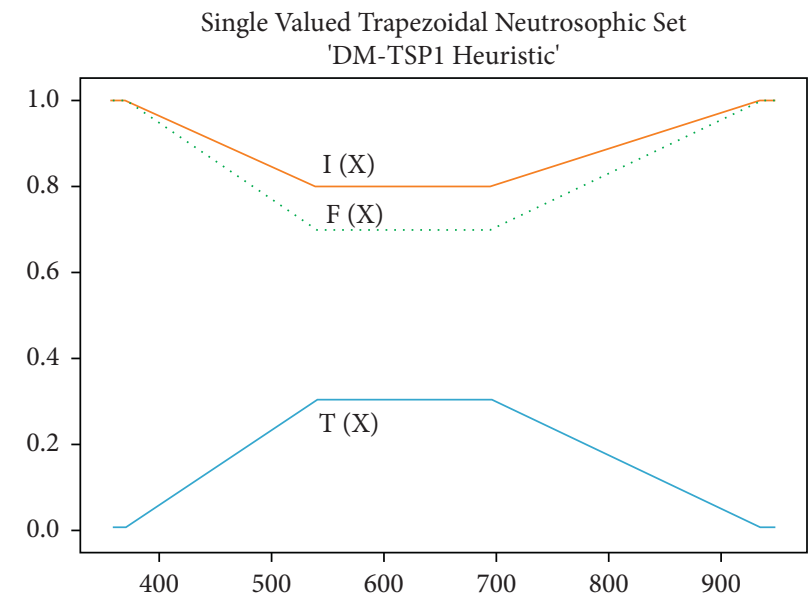

FIgURE 11: The minimal total single-valued trapezoidal neutrosophic cost using the DM-TP1 heuristic.

Consequently, the degree of falsity for the minimal single-valued trapezoidal neutrosophic number cost is depicted by

$$
\lambda(X)= \begin{cases}\frac{543-x+(x-370) \times 0.7}{543-370}, & 370 \leq x \leq 543, \\ 0.7, & 543 \leq x \leq 694, \\ \frac{x-694+(938-x) \times 0.7}{938-694}, & 694 \leq x \leq 938, \\ 0, & \text { otherwise. }\end{cases}
$$

Based on the above result generated by the DM-TP1 heuristic, the decision maker can schedule the transportation network diagram plan.

\section{Conclusion}

The transportation problem is a special type in supply chain management where the aim is to minimize the total transportation cost of shipping products from sources to destinations. This paper addresses a transportation problem model under single-valued trapezoidal neutrosophic environment. To solve this problem, the novel Dhouib-MatrixTP1 heuristic is enhanced at first with a score function in order to ensure the defuzzification of the neutrosophic trapezoidal fuzzy numbers into crisp numbers and at second with a new metric function (Average-Min) in order to drive the selection process.

Stepwise numerical applications are used to explain and to prove the performance of the proposed DM-TP1 heuristic. It also shows the effect of using the Average and the Min descriptive statistical metrics on the accuracy of the decision made. Further research will focus on the application 
of the Dhouib-Matrix-TP1 to solve the multiobjective transportation problem in uncertain environment.

\section{Data Availability}

All data used to support the findings of the study are included within the article.

\section{Conflicts of Interest}

The authors declare that they have no conflicts of interest.

\section{References}

[1] F. Smarandache, A Unifying Field in Logics: Neutrosophic Logic. Neutrosophy, Neutrosophic Set, Neutrosophic Probability and Statistics, InfoLearnQuest, Philadelphia, PA, USA, 6th edition, 2007.

[2] N. A. Ibrahem, H. Elghareeb, F. F. Farahat, and A. AboElfotouh, "Comparative mathematical model for predicting of financial loans default using altman Z-score and neutrosophic AHP methods," Neutrosophic Sets and Systems, vol. 43, pp. 24-43, 2021.

[3] N. S. M. Assarudeen, J. V. Sudhakar, and S. Balakrishnan, “A new approach for solving assignment problem in neutrosophic environment by zero suffix method," International Journal of Current Advanced Research, vol. 7, no. 1, pp. 129-132, 2018.

[4] K. Selvakumari and S. Subasri, "Comparative analysis of fuzzy, intuitionistic and neutrosophic assignment problem using nonagonal fuzzy number," European Journal of Molecular \& Clinical Medicine, vol. 7, no. 2, pp. 5099-5109, 2020.

[5] K. Radhika and K. A. Prakash, "Ranking of pentagonal neutrosophic numbers and its applications to solve assignment problem," Neutrosophic Sets and Systems, vol. 35, pp. 465-477, 2020.

[6] S. Kar, K. Basu, and S. Mukherjee, "Solution of multi-criteria assignment problem using neutrosophic set theory," Neutrosophic Sets and Systems, vol. 10, pp. 31-38, 2015.

[7] H. A. Khalifa and P. Kumar, "A novel method for solving assignment problem by using interval-valued trapezoidal neutrosophic number," Neutrosophic Sets and Systems, vol. 36, pp. 24-36, 2020.

[8] S. K. Prabha and S. Vimala, "Neutrosophic assignment problem via $\mathrm{BnB}$ algorithm," Trends in Mathematics, Advances in Algebra and Analysis, pp. 323-330, 2019.

[9] S. Subasri and K. Selvakumari, "Neutrosophic travelling salesman problem in trapezoidal fuzzy number using Branch and bound technique," Journal of Physics: Conference Series, vol. 1362, Article ID 012098, 2019.

[10] S. Subasri and K. Selvakumari, "Solving neutrosophic travelling salesaman problem in triangular fuzzy number using ones assignment method," Eurasian Journal of Analytical Chemistry, vol. 13, pp. 285-291, 2018.

[11] K. P. Sikkannanl and V. Shanmugavel, "Unraveling neutrosophic transportation problem using costs mean and Complete contingency cost table," Neutrosophic Sets and Systems, vol. 29, pp. 165-173, 2019.

[12] J. Ye, "Trapezoidal fuzzy neutrosophic set and its application to multiple attribute decision making," Soft Computing, vol. 26, no. 5, pp. 1157-1166, 2015.

[13] P. Biswas, S. Pramanik, and C. B. Giri, "Aggregation of triangular fuzzy neutrosophic set information and its application to multi attribute decision making," Neutrosophic Sets and Systems, vol. 12, pp. 20-40, 2016.

[14] S. Dhouib, "Optimization of travelling salesman problem on single valued triangular neutrosophic number using dhouibmatrix-TSP1 heuristic," International Journal of Engineering, vol. 34, no. 12, pp. 1-6, 2021.

[15] A. Thamaraiselvi and R. Santhi, "A new approach for optimization of real life transportation problem in neutrosophic environment," Mathematical Problems in Engineering, vol. 2016, Article ID 5950747, 9 pages, 2016.

[16] A. Singh, A. Kumar, and S. S. Appadoo, "Modified approach for optimization of real life transportation problem in neutrosophic environment," Mathematical Problems in Engineering, vol. 2017, Article ID 2139791, 9 pages, 2017.

[17] S. Broumi, A. Bakali, M. Talea, F. Smarandache, and L. Vladareanu, "Shortest path problem under triangular fuzzy neutrosophic information," in Proceedings of the 2016 10th International Conference on Software, Knowledge, Information Management \& Applications (SKIMA), Chengdu, China, December 2016.

[18] F. Karaaslan and K. Hayat, "Some new operations on singlevalued neutrosophic matrices and their applications in multicriteria group decision making," Applied Intelligence, vol. 48, no. 12, pp. 4594-4614, 2018.

[19] G. Muhiuddin, "p-ideals of BCI-algebras based on neutrosophic N -structures," Journal of Intelligent and Fuzzy Systems, vol. 40, no. 1, pp. 1097-1105, 2021.

[20] S. Ashraf, S. Abdullah, S. Zeng, H. Jin, and F. Ghani, "Fuzzy decision support modeling for hydrogen power plant selection based on single valued neutrosophic sine trigonometric aggregation operators," Symmetry, vol. 12, no. 2, pp. 1-27, 2020.

[21] L. F. Hitchcock, "The distribution of a product from several sources to numerous localities," Journal of Mathematics and Physics, vol. 20, no. 2, pp. 224-230, 1941.

[22] A. Charnes and W. W. Cooper, "The stepping Stone method of explaining linear programming calculations in transportation problems," Management Science, vol. 1, no. 1, pp. 49-69, 1954.

[23] G. B. Dantzig, Linear Programming and Extensions, Princeton University Press, Princeton, NJ, USA, 1963.

[24] E. Shell, “"Distribution of a product by several properties”, directorate of management analysis," Proceedings of the Second Symposium in Linear Programming, vol. 2, pp. 615-642, 1955.

[25] K. B. Haley, "New methods in mathematical programmingThe solid transportation problem," Operations Research, vol. 10, no. 4, pp. 448-463, 1962.

[26] P. Pandian and G. Natarajan, "A new algorithm for finding a fuzzy optimal solution for fuzzy transportation problems," Applied Mathematical Sciences, vol. 4, no. 2, pp. 79-90, 2010.

[27] P. S. Kumar, "PSK method for solving type-1 and type-3 fuzzy transportation problems," International Journal of Fuzzy System Applications, vol. 5, no. 4, pp. 367-392, 2016.

[28] A. S. Mhaske and K. L. Bondar, "Fuzzy transportation problem by using triangular, pentagonal and heptagonal fuzzy numbers with Lagrange's polynomial to approximate fuzzy cost for nonagon and hendecagon," International Journal of Fuzzy System Applications, vol. 9, no. 1, 2020.

[29] S. D. Dinagar and K. Thiripurasundari, "A navel method for solving fuzzy transportation problem involving intuitionistic trapezoidal fuzzy numbers," International Journal of Current Research, vol. 6, no. 6, pp. 7038-7041, 2014.

[30] K. P. Sikkannan and V. Shanmugavel, "Sorting out fuzzy transportation problems via ECCT and standard deviation," 
International Journal of Operations Research and Information Systems, vol. 12, no. 2, 2021.

[31] H. Wang, F. Smarandache, Y. Q. Zhang, and R. Sunderraman, "Single valued neutrosophic sets," Multispace and Multistructure, vol. 4, pp. 410-413, 2010.

[32] S. Dhouib, "A new column-row method for traveling salesman problem: the dhouib-matrix-TSP1," International Journal of Recent Engineering Science, vol. 8, no. 1, pp. 6-10, 2021.

[33] Sa. Dhouib and S. Dhouib, "Optimizing the trapezoidal fuzzy travelling salesman problem through dhouib-matrix-TSP1 method based on magnitude technique," International Journal of Scientific Research in Mathematical and Statistical Sciences, vol. 8, no. 2, pp. 1-4, 2021.

[34] M. Miledi, S. Dhouib, and T. Loukil, "Dhouib-Matrix-TSP1 method to optimize octagonal fuzzy travelling salesman problem using $\alpha$-cut technique," International Journal of Computer and Information Technology, vol. 10, no. 3, pp. 130-133, 2021.

[35] S. Dhouib, "Minimizing the total distance for the supply Chain problem using dhouib-matrix-TSP2 method," International Journal of Advanced Research in Engineering \& Technology, vol. 12, no. 5, pp. 1-12, 2021.

[36] S. Dhouib, "A novel heuristic for the transportation problem: dhouib-matrix-TP1," International Journal of Recent Engineering Science, vol. 8, no. 4, pp. 1-5, 2021. 